\title{
Effet de l'alimentation calcique séparée sur les performances de production de la poule LHOMAN BROWN sous climat tropical humide
}

\author{
Mantsanga² H. B., Amona² I., Banga-Mboko H., ${ }^{1-2^{\star}}$ Bakana ${ }^{1}$ M.A, et Adzona² P.P, \\ ${ }^{1}$ Institut National de Recherche Agronomique, Brazzaville, Congo. \\ 2Ecole Nationale Supérieure d'Agronomie et de Foresterie, Université Marien Ngouabi, Brazzaville, Congo, BP. 69 Congo \\ Brazzaville \\ *Auteur correspondant : henribangamboko@yahoo.fr \\ Tel. (+242) $066851476 /(+242) 055778025$
}

Original submitted in on $18^{\text {th }}$ June 2015. Published online at www.m.elewa.org on $31^{\text {st }}$ January 2016 http://dx.doi.org/10.4314/jab.v97i1.6

\section{RÉSUMÉ}

Object: L'étude envisagée, se propose de vérifier la réponse de la souche Lhoman Brown soumise à l'alimentation calcique séparée. Elle s'inscrit dans le contexte de la sécurité alimentaire, la lutte contre la pauvreté et les changements climatiques sous climat tropical humide.

Matériels et méthodes : L'étude a été menée au Congo-Brazzaville, sur deux bandes de poules pondeuses qui ont préalablement été nourries lors de la phase pré-ponte par un aliment complet de 2856,56 kcal/kg d'EM et $15,55 \%$ de protéine. Le premier groupe (témoin) a reçu un aliment complet alors que le second (traité), un aliment appauvri en calcium. Le calibre des œufs, la qualité des coquilles et le coût de production de l'œuf sont les principales variables prises en considération lors de cette étude.

Résultats : II en ressort que les poules du lot traité, présentent $41,84 \%$ contre $36,18 \%$, des œufs de calibre moyen et le poids moyen des coquilles est de l'ordre de 5,90 $\pm 0,95 \mathrm{~g}$ contre $5,42 \pm 0,64 \mathrm{~g}$. Le coût de production des œufs du lot traité, étant réduit à un niveau de $-14,94 \%$.

Conclusion et application des résultats : Les arguments qui sont en faveur de l'alimentation calcique séparée ont été mis en exergue. Ainsi, l'alimentation calcique séparée permet d'améliorer la qualité des produits et la rentabilité de l'élevage des poules.

Mots clés : alimentation, Lhoman Brown, performances de production, climat tropical humide, CongoBrazzaville.

\section{ABSTRACT}

Effect of the separate calcium feeding on the performances of LHOMAN BROWN laying hens under tropical climate

Objective: in hot climates, the performances of laying hens are compromised by heat stress. The separate calcium feeding has been suggested as one of the ways to reduce the harmful effect of heat on the productivity of hens under hot climate. The aim of this work was to evaluate the response of the Lhoman Brown strain subjected to a separate calcium feeding. 
Materials and methods: the study was conducted on a sample of one- day laying hens fed firstly with a complete food containing $2856.56 \mathrm{kcal} / \mathrm{kg} \mathrm{ME}$ and $15.55 \%$ protein still the age of 25 weeks. Secondly, the hens have been separated into two groups. The first group (control) received a complete feed and the second (Treatment) received a diet depleted of calcium and a calcium source distributed separately. The hens were compared on the variables related on the egg size, egg quality and the cost of egg production. The results showed that the treatment improved significantly egg size $(41,85 \%)$, egg shelll $(5,99 \mathrm{~g})$. Also, the treatment reduced significantly the cost of $14,94 \%$ egg productions.

Conclusion and application of results: the study highlighted arguments that separate calcium feeding is an alternative way to improve egg quality, egg size and the profitability in small livestock farming in hot climates, also in order to meet the needs of the market.

Keywords: feeding, Lhoman Brown, egg, Calcium, shell, egg size, egg cost

\section{INTRODUCTION}

La volaille occupe une place importante au Congo, aussi bien dans la production, les importations que dans la consommation des produits carnés. En effet l'élevage de la volaille est la plus importante activité de la production animale (FAO, 2009). Dans l'importation des produits animaux, la viande de volaille représente également la part la plus importante, évaluée entre 70 et $75 \%$ en volume et 40 à $50 \%$ de la valeur des importations (FAO, 2009). La dépendance du Pays vis-à-vis de l'extérieur en œufs de table est $86,7 \%$. La production nationale en œufs de table est faible et ne représente que $10 \%$ de la consommation (FAO, 2009). L'aviculture congolaise est donc confrontée à un certain nombre de problèmes dont les plus importants sont l'alimentation (FAO, 2009) et la chaleur (BangaMboko et al., 2003, 2007). La pénurie de l'aliment de bétail en terme de qualité et de coût constitue un frein au développement de l'élevage des monogastriques au Congo. En élevage des poules pondeuses, l'intensité de ponte, le calibre et la qualité des œufs sont les clés de voûte de la rentabilité de l'activité fermière. De nos jours, plusieurs sujets épineux font l'objet des débats à l'échelle mondiale, notamment, l'effet de certains facteurs sur la rentabilité en zone tropicale. En climat chaud, ces éléments de rentabilité sont compromis par la chaleur qui a des effets néfastes sur les performances de production de poules pondeuses (Picard et al., 1993, Joly, 1999 ; BangaMboko et al., 2007 ; Brou et al., 2012). Dans ce milieu, les baisses de croissance et les diminutions de performances ont pour conséquences la diminution des consommations d'aliment, lorsque les animaux sont dans l'incapacité de réguler leur température corporelle (Banga-Mboko et al., 2007). Ainsi, face à cette situation, plusieurs solutions en vue de limiter les effets d'un stress thermique ont été proposées, entre autres l'alimentation calcique séparée (Banga-Mboko et al., 2007). Cette technique a été suggérée pour limiter les effets néfastes de la température élevée sur la productivité de la poule pondeuse en climat chaud (Mongin et Sauveur ,1975; Cabrera et al., 1982 ; Abdelatif et al., 1986 ; Uzu, 1989 ; Bordas et Minvielle 1997). Les effets pratiques de ce type d'apport calcique sur la production et la qualité des œufs ont été évalués par plusieurs expériences (Banga-Mboko et al., 2003 ; Banga-Mboko et al., 2007). Cette pratique a aussi pour effet de mieux répondre aux besoins spécifiques en calcium de la poule selon qu'il y a formation de l'œuf ou pas car, plusieurs auteurs ont rapporté par leurs travaux, l'aptitude des poules à sélectionner naturellement un régime alimentaire (Mongin et Sauveur, 1975 ; Banga-Mboko et al., 2003). II a été démontré dans les conditions du milieu chaud que la distribution d'un aliment complet chez la poule en ponte constitue un gaspillage ou une surconsommation de l'aliment chez la poule qui recherche spécifiquement du calcium (Bordas et Minveille, 1997). En revanche, l'addition de coquilles d'huîtres ou d'autres sources calciques à un régime déjà riche en calcium est inefficace de ce point de vue (Bordas et Minveille, 1997). En Afrique et principalement au Congo, le prix d'achat d'un œuf de table galope du jour au jour. II est passé de 25 à 35 francs CFA et de 35 francs CFA à 125francs CFA puis de 125 francs à 150 francs CFA de nos jours. 
Cette élévation est due par le fait que la plupart des matières premières utilisées dans la fabrication des aliments sont importées. Corrélativement, le $\mathrm{kg}$ d'aliment est passé de 150 francs CFA à 410 francs CFA. Ainsi, le plateau de 30 œufs vendu à 1200 et 1500 FCFA au Cameroun, 1500 et 1800 FCFA au Sénégal, 1000 et 1500 FCFA au Mali et 25002750FCFA en RCA (OFIVAL, 2005), est vendu à 3750 au Congo- Brazzaville. La maîtrise du calibre de l'œuf et de la qualité de la coquille est un critère extrêmement important pour le producteur d'œuf. En effet, la mise en place en vente doit faire l'objet d'un bon compromis entre le besoin du marché et la meilleure qualité de coquille et de l'œuf tout entier (Joly, 1999 ; Akouango, 2014). Par ailleurs, un poids d'œuf trop faible entraîne souvent une réduction de la rémunération et un poids d'œuf trop élevé entraîne une augmentation du taux de déclassement en fin de ponte. Le sélectionneur recherche le

\section{MATERIEL ET METHODES}

Présentation de la zone d'étude : L'étude a été réalisée à Brazzaville, en République du Congo dans la ferme expérimentale du Centre de Recherches Vétérinaires et Zootechniques (C.R.V.Z) situé dans l'arrondissement 2 Bacongo. Le climat de Brazzaville est tropical humide de type bas-congolais (Samba et Moundza, 2007). II est caractérisé par deux saisons: une saison de pluies d'octobre à mai avec un fléchissement en janvier et une saison sèche de juin à septembre. La saison des pluies commence généralement entre le 11 et le 16 Septembre pour terminer entre le 14 et le 20 mai. Ces dernières années, une tendance tardive de l'arrivée du début de la saison de pluies est constatée (Mialoundama et Ndinga, 2006).

Caractéristiques de la souche de poule élevée : Les poules élevées appartiennent à la souche commerciale Lohmann Brown, de hautes performances zootechniques, sélectionnée en Allemagne. Ces poules sont réputées sur le marché, avec des performances durables: pic de ponte élevé, persistance de ponte, coloration et qualité de coquille. Les caractéristiques de ces pondeuses contribuent à atteindre un niveau optimal de production dans des conditions d'élevage variées. Aux œufs de couleur de la coquille roux agréable, elles supportent mieux le climat tropical et s'alimente sans aucun risque d'engraissement. Son taux de viabilité est de $97-98 \%$ en période d'élevage et $94-96 \%$ en période de ponte. La poule a un pic de ponte qui varie entre 92 à $94 \%$, pour un meilleur compromis (Joly, 1999). Ainsi, dans les pays chauds, la chaleur limite d'une manière importante l'ingestion de l'aliment dont toutes les composantes sont presque importées. Cependant, malgré une faible industrialisation de l'agriculture en Afrique, les sous-produits agricoles et agro industriels locaux inventoriés et qui peuvent contribuer à améliorer la compétitivité de filières avicoles sont peu étudiés et peu valorisés ( $F A O$, 2008). L'objectif de cette étude est d'évaluer l'effet de l'ACS composée de matières premières locales sur les performances de ponte et le coût de production de l'œuf. La présente étude évalue pour la première fois le coût de production de l'œuf en ACS et l'applicabilité de l'ACS à partir d'un aliment composé des matières premières locales. Aussi, ce travail évalue pour la première fois la réponse de la souche lohman à ce mode d'alimentation en climat chaud.

nombre d'œufs par poule démarrée de 350 - 315 en 12 mois de ponte. Le poids moyen de l'œuf en 12 mois de ponte en compris entre $63,5-64,5 \mathrm{~g}$ et l'indice de consommation est de 2,1 -2,2 Kg/Kg (Aviforum, 1999). Phase pré-expérimentale (élevage des poulettes) : A l'arrivée, les poussins ont été élevés au sol sur la litière et nourris avec un aliment démarrage jusqu'à 8 semaines. Ensuite, ils ont été transférés dans le local des poulettes de $14 \mathrm{~m}^{2}$ de surface. A partir de la gème jusqu'à la 18 ième semaine, ces poussins ont été nourris en utilisant un aliment poulette contenant $2856,56 \mathrm{Kcal} / \mathrm{kg}$ et $15,55 \%$ MAT.

Phase expérimentale (élevage des pondeuses)

Animaux et conditions expérimentales : A partir de la $19^{\text {ième }}$ semaine, les pondeuses ont été séparées de manière aléatoire en deux lots de 50 poules chacun. Ensuite, elles ont été transférées dans deux nouveaux compartiments. Tout comme des poulettes, les poules ont été élevées au sol sur la litière qui est constituée du copeau de bois, dans les mêmes conditions d'humidité, de ventilation et de température. Les moyennes de températures étaient de $28,98^{\circ} \mathrm{C}$ le matin et $30,04^{\circ} \mathrm{C}$ le soir durant toute la période expérimentale. Les mesures de prophylaxie sanitaire (hygiène, vide sanitaire) et de prophylaxie médicale ont été mises en application pour éviter et prévenir l'apparition d'éventuelles pathologies.

Régimes alimentaires : Le premier lot (témoin) a été constitué des poules soumises à un régime alimentaire 


\section{Mantsanga et al. J. Appl. Biosci. 2016 Effet de l'alimentation calcique séparée sur les performances de production de la poule LHOMAN BROWN sous climat tropical humide}

conventionnel (figure 1) et le second lot appelé lot des poules traitées (figure 2) a été constitué des poules soumises à un régime alimentaire expérimental. Chaque compartiment était équipé de mangeoires et abreuvoirs. Les poules du lot traité ont été soumises à un régime alimentaire constitué d'un aliment de base appauvri en calcium $(0,58 \%$ de calcium) servi dans une première mangeoire et le calcaire séparément dans une seconde

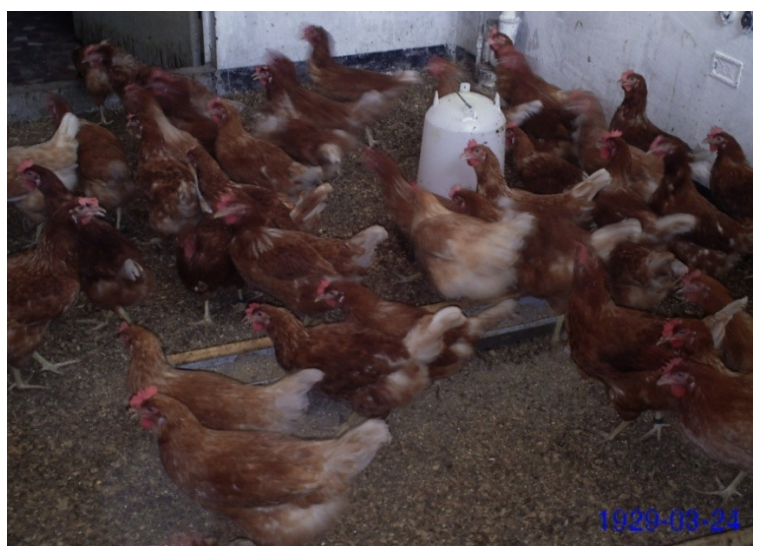

Figure 1. Lot de poules témoin soumis à l'alimentation complète mangeoire. Par contre, le lot des témoins était soumis à un aliment composé complet contenant $3,55 \%$ de calcium et distribué dans une seule mangeoire. L'aliment a été distribué ad libitum dans les deux lots de poules durant toute la période expérimentale. Les refus d'aliment et d'eau ont été pesés quotidiennement dans chaque lot de poules.

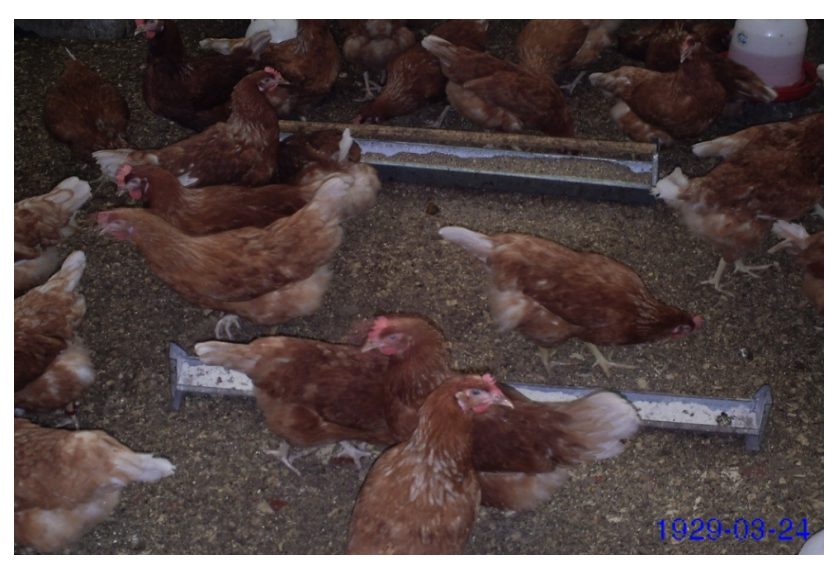

Figure 2 : Lot de poules traitées soumises à l'alimentation calcique séparée autour d'une mangeoire contenant de l'aliment sans calcaire d'un côté et de l'autre côté une mangeoire contenant du calcaire

La composition et l'analyse chimique des deux régimes alimentaires sont données dans le tableau 1.

Tableau 1 : Composition et analyse chimique des rations alimentaires

\begin{tabular}{|l|l|l|}
\hline Composition & Aliment complet (lot témoin) & Alimentation calcique séparée (lot traité) \\
\hline Maïs & 35 & 37,5 \\
\hline Drêche & 20 & 21,25 \\
\hline Farine de manioc & 13,5 & 14,25 \\
\hline Tourteau d'arachide & 5 & 5,25 \\
\hline Tourteau de soja & 4 & 4,25 \\
\hline Farine de poisson & 8 & 8,5 \\
\hline Farine de niébé & 8 & 8,5 \\
\hline Calcaire & 6 & 0 \\
\hline Vitamine & 0,05 & 0,05 \\
\hline Sel & 0,45 & 0,045 \\
\hline Total & 100 & 100 \\
\hline Analyse chimique & \multicolumn{2}{|l|}{} \\
\hline Calcium (\%) & 3,55 & 0,58 \\
\hline Protéines brutes (\%) & 18,36 & 19,49 \\
\hline Énergie métabolisable (Kcal) & 2812,68 & 2993,99 \\
\hline Ratio EM / PB & 153,19 & 153,61 \\
\hline
\end{tabular}




\section{Mantsanga et al. J. Appl. Biosci. 2016 Effet de l'alimentation calcique séparée sur les performances de production de la poule LHOMAN BROWN sous climat tropical humide}

Variables mesurées.

Calibre d'œufs : Les œufs de chaque lot de poules qui sont collectés durant une période de 10 semaines, ont été pesés et classés en catégories (petit, moyen, gros et très gros) selon la classification recommandée (tableau 2).

Tableau 2. Classification des œufs en fonction du calibre. (Anonyme, 2013).

\begin{tabular}{|l|l|l|l|l|}
\hline Calibre & S (petit) & M & L & XL \\
\hline Poids & $53 \mathrm{~g}$ & 53 à $63 \mathrm{~g}$ & $63-73 \mathrm{~g}$ & $73 \mathrm{~g}$ \\
\hline
\end{tabular}

Qualité de la coquille : Un échantillon de 30 œufs était retenu dans chaque lot de poules pour évaluer la qualité de la coquille notamment le poids, l'épaisseur et le pourcentage de la coquille. Chaque œuf était d'abord pesé puis cassé. La coquille étant ensuite nettoyée et pesée. L'épaisseur de la coquille (en $\mathrm{mm}$ ) a été déterminée à l'aide d'un pied à coulisse sur 3 fragments qui sont prélevés au niveau des deux bouts et au centre de la coquille.

Tableau 3. Tarif des matières premières

\begin{tabular}{|l|l|}
\hline Matières premières & Prix du Kg (FCFA) \\
\hline Maïs & 215 \\
\hline Drêche & 20 \\
\hline Farine de manioc & 40 \\
\hline Arachide grain & 300 \\
\hline Tourteau de soja & 810 \\
\hline Farine de poisson & 250 \\
\hline Niébé grain & 300 \\
\hline Calcaire & 140 \\
\hline Vitamine & 24000 \\
\hline Sel & 600 \\
\hline
\end{tabular}

Analyse et traitement statistique : Les moyennes des deux régimes alimentaires ont été comparées par le logiciel statview en utilisant le t-test. A cet effet, deux

\section{RÉSULTATS}

Les résultats relatifs aux paramètres mesurés sont présentés au figure 1 et dans les tableaux 4,5 .

Effet de l'alimentation calcique séparée sur le calibre des œufs: Les poules du lot traité ont donné plus des œufs de calibre standard pesant $53-63 \mathrm{~g}(41,84 \%)$ et les
Coût de production de l'œuf : Le paramètre cout de production de l'œuf a été déterminé suivant le schéma ci-après :

$>\quad$ La détermination du prix d'un $\mathrm{kg}$ d'aliment de chaque régime alimentaire ;

$>\quad$ La détermination du coût de la consommation totale d'aliment pour chaque lot de poules.

La détermination du coût de production de l'œuf pour la consommation totale d'aliment. Le prix du $\mathrm{kg}$ de chaque matière première est donné au tableau 3. moyennes étaient statistiquement différentes quand après transformation la valeur de $\mathrm{P}$ était inférieure à 0,05 .

œufs de $63-73 \mathrm{~g}(1,08 \%)$ contre $36,18 \%$ des œufs de $53-63 \mathrm{~g}$ et $1,02 \%$ de $63-73 \mathrm{~g}$ pour les poules du lot témoin (Figure 3). Aucun œuf pesant plus de $73 \mathrm{~g}$ n'a été obtenu dans les deux groupes. 

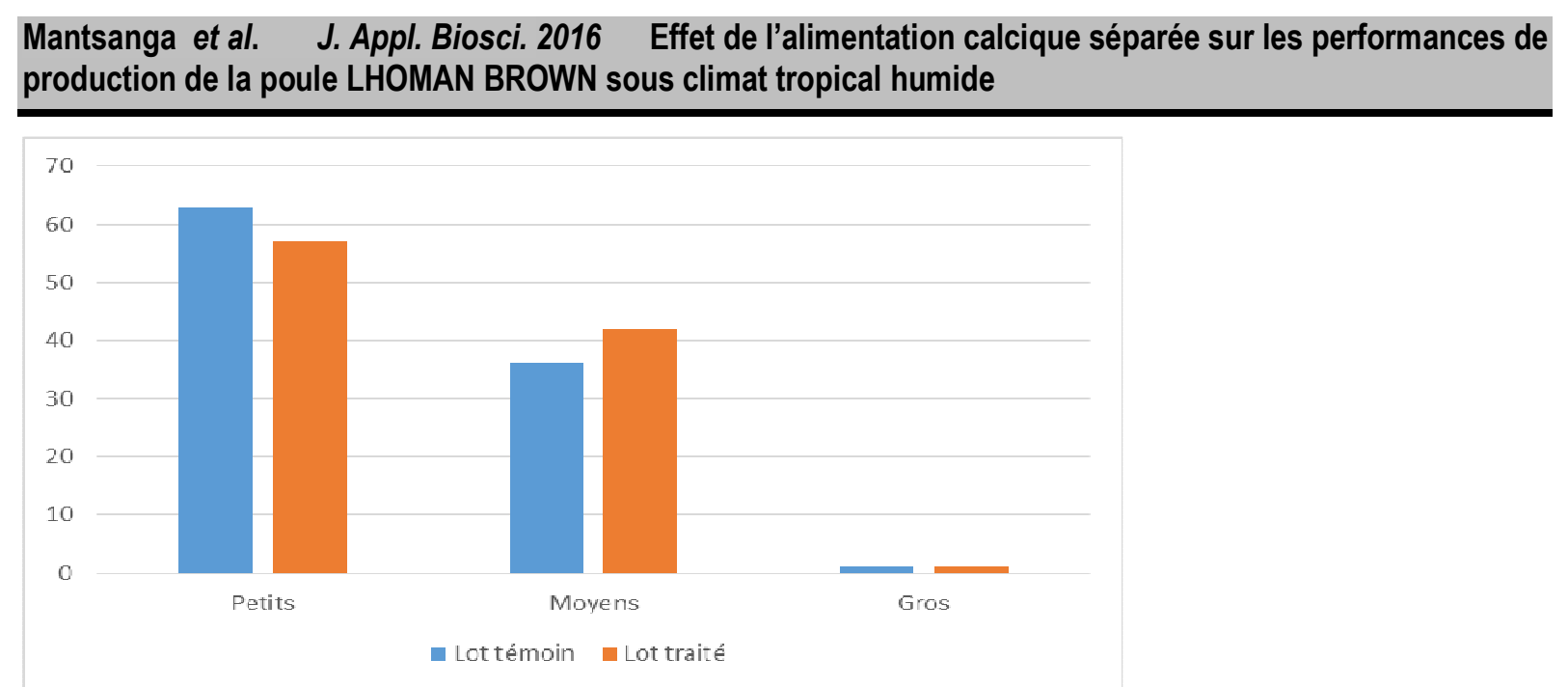

Figure 3 : Effet de l'alimentation calcique séparée (lot complet et lot traité) sur le calibre des œufs.

Les résultats sur le calibre montrent que le traitement a diminué significativement le pourcentage de petits œufs et a augmenté les œufs de poids moyen et les gros œufs. Effet de l'alimentation calcique séparée sur la qualité de la coquille: Les poules en alimentation calcique séparée ont donné les œufs dont le poids et l'épaisseur de la coquille sont largement supérieurs aux résultats enregistrés chez les poules soumises à l'alimentation complète (tableau 5).

Tableau 5. Effet de l'alimentation calcique séparée sur la qualité de la coquille

\begin{tabular}{|l|l|l|l|}
\hline Variables & Lot Témoin & Lot Traité & Signification de la valeur de $\mathbf{P}$ \\
\hline Poids moyen de l'œuf(g) & $52,83 \pm 4,48$ & $54,67 \pm 5,45$ & S \\
\hline Poids de la coquille $(\mathrm{g})$ & $5,42 \pm 0,64$ & $5,90 \pm 0,95$ & $\mathrm{~S}$ \\
\hline Épaisseur du petit bout $(\mathrm{mm})$ & $0,32 \pm 0,03$ & $0,43 \pm 0,10$ & $\mathrm{~S}$ \\
\hline Épaisseur du milieu de la coquille $(\mathrm{mm})$ & $0,31 \pm 0,03$ & $0,36 \pm 0,07$ & $\mathrm{~S}$ \\
\hline Épaisseur du gros bout $(\mathrm{mm})$ & $0,27 \pm 0,03$ & $0,35 \pm 0,09$ & $\mathrm{~S}$ \\
\hline Pourcentage de la coquille & $10 \pm 0,17$ & $11 \pm 0,14$ & $\mathrm{~S}$ \\
\hline
\end{tabular}

$S=$ significative $(p<0,05) ; N S=$ Non significative $(P>0,05)$

Effet de l'alimentation calcique séparée sur le coût de production de l'œuf : Le coût de production de l'œuf en alimentation calcique séparée (ACS) est nettement inférieur au coût de production de l'œuf du régime alimentaire ordinaire (tableau 6).

Tableau 6. Effet de l'alimentation calcique séparée sur le coût de production de l'œuf

\begin{tabular}{|l|l|l|}
\hline Variables & $\begin{array}{l}\text { Alimentation } \\
\text { complète }\end{array}$ & $\begin{array}{l}\text { Alimentation calcique } \\
\text { séparée }\end{array}$ \\
\hline $\begin{array}{l}\text { Coût de la charge alimentaire de chaque régime } \\
\text { (FCFA) pour produire un œuf }\end{array}$ & 52 & 39 \\
\hline Coût des autres charges impliquées pour la production d'un œuf & 35 & 35 \\
\hline Coût de production (CP) de l'œuf avec toutes les charges (FCFA) & 87 & 74 \\
\hline Prix de vente (+25\% du CP) & 109 & 93 \\
\hline
\end{tabular}

\section{DISCUSSION}

De nombreuses études (Cabrera et al., 1982 ; Abdelatif et al., 1986 ; Banga-Mboko et al., 2001,2003 et 2007 ) ont été menées sur l'effet de l'alimentation calcique séparée pour améliorer les performances de production de la poule pondeuse. En effet, l'aliment utilisé dans la présente étude a été fabriqué à partir des matières premières locales disponibles sur le marché, suggérant l'hypothèse de valorisation des ressources locales. Les 


\section{Mantsanga et al. J. Appl. Biosci. 2016 Effet de l'alimentation calcique séparée sur les performances de production de la poule LHOMAN BROWN sous climat tropical humide}

résultats ont monté que les œufs collectés appartiennent à 3 catégories (petite, moyenne et large) sur 4 selon le classement recommandé (Anonyme 2013). L'étude a montré que le traitement a amélioré le nombre d'œufs pondu et leur poids. D'après la littérature, l'œuf le plus couramment utilisé pour la cuisine est celui appartenant au poids moyen entre 53 et $63 \mathrm{~g}$ (Anonyme, 2013). Dans ce contexte, l'alimentation calcique séparée a augmenté de $5,68 \%$ le nombre des œufs de calibre moyen et a réduit de $-5,74 \%$ le nombre des œufs de calibre petit, suggérant ainsi l'hypothèse d'une amélioration de la qualité de l'œuf notamment son poids par l'apport d'un régime calcique séparé (Banga-Mboko et al., 2007). L'effet de la variation des températures sur le poids et la taille des œufs a été révélé par plusieurs auteurs (Sauveur et Picard, 1990; Brou et al., 2012). Les résultats ont montré que le poids moyen de l'œuf en alimentation calcique séparé a été plus élevé qu'en alimentation complète. Cette amélioration des performances de ponte peut s'expliquer par le fait que durant les périodes chaudes les poules traitées maintiennent leur température corporelle, ce qui n'influe pas sur la formation de l'œuf. Dans le même contexte, Brou et al., (2012) révèlent une hétérogénéité sur la taille et le poids des œufs pondus dans la journée due à la variation de la températion journalière chez les poules soumises en alimentation ordinaire. Selon Brou et al., (2012), les gros œufs sont pondus à des heures de basses températures alors que pendant les heures chaudes de la journée les œufs pondus sont de petite taille. La fragilité de la coquille est un facteur limitant de la production des œufs. En effet, le traitement a amélioré significativement $(p<0,05)$ la qualité de la coquille notamment le poids de la coquille $(+16 \%)$, l'épaisseur moyen du petit bout $(+33 \%)$, l'épaisseur moyen du gros bout $(+17 \%)$ et du centre de la coquille $(27 \%)$. Ces résultats sont en accord avec la plupart des travaux sur l'alimentation calcique séparée (Cabrera et al., 1982 ; Abdelatif et al.,1986 ; Banga Mboko et al., 2003 ; Banga Mboko et al., 2007). D'autres auteurs (Akouango, 2014) relèvent la variabilité de la qualité de l'œuf à différentes phases de ponte. Le coût de production de l'œuf obtenu a connu une réduction de $-14,94 \%$ en ACS. Ce résultat

\section{CONCLUSION}

Cette étude avait pour objectif d'évaluer la réponse à l'ACS, de la souche Lohman Brown. Les résultats obtenus montrent que l'ACS est un mode d'alimentation valorisable en élevage dans les pays à climat tropical et humide comme le Congo-Brazzaville. Ce mode d'alimentation cacique séparée permet de réduire les est largement inférieur à celui obtenu par la FAO (2009) à l'issue d'une étude sur la cartographie de la filière avicole au Congo Brazzaville. Dans cette étude, le coût de production d'un œuf de table au Congo Brazzaville s'élève à 91 ,32 FCFA (FAO, 2009). En effet, pour un prix de vente d'un œuf en détail à 125 FCFA (FAO, 2009), la marge bénéficiaire d'un œuf de table est de 33,68 FCFA, largement inférieure à celle obtenue par l'utilisation de l'alimentation calcique séparée qui s'élève à 32 FCFA. Les prix de vente de 109 et 93 FCFA respectivement pour les poules soumises à l'alimentation ordinaire et celles soumises à l'alimentation calcique séparées sont inférieurs au prix de vente (125 FCFA) d'un œuf sur le marché Congolais. L'alimentation calcique séparée a engendré d'une part, une baisse de $-14,67 \%$ le prix de vente par rapport au prix de vente de l'œuf produit par l'aliment ordinaire et d'autre part, une baisse de $-25,6 \%$ du prix de vente de l'œuf du marché national. Ces résultats montrent que l'œuf produit en alimentation calcique séparée procure plus de bénéfice. II est donc plus économique que l'œuf produit en alimentation ordinaire. Cette étude montre qu'un apport en aliment calcique séparé favorise une augmentation du calibre des $œ u f s$ et leur production à un cout moindre. Ce faible coût de production suppose que la masse d'œufs a été élevée chez les poules traitées pour une consommation d'aliment réduite. D'après ces résultats le plateau de 30 œufs en alimentation calcique séparée peut être vendu au Congo à un coût relativement identique à celui de la RCA, supérieur au prix du Sénégal et largement supérieur au prix du Cameroun et Mali (OFIVAL, 2005). L'évaluation du cout de production présente un grand intérêt dans le sens où il permet à l'éleveur ou à l'investisseur d'avoir une idée précise sur les données économiques en matière de cet élevage. Ces résultats suggèrent une valorisation des matières premières locales dans la fabrication de l'aliment de bétail. Sur l'ensemble des variables mesurées, les poules Lohmann ont donné une réponse favorable à l'alimentation calcique séparée, indiquant l'expression d'un appétit spécifique en calcium. Cette réponse rappelle celle obtenue chez les poules Shaver dans les mêmes conditions du Congo Brazzaville (Banga-Mboko et al., 2007).

effets néfastes des températures élevées sur la productivité des poules pondeuses en milieu tropical. II est impérieux de poursuivre cette étude en mettant l'accent sur les conditions d'élevage pour améliorer la production et la qualité des œufs. Les rations utilisées dans cette étude ont été fabriquées à partir des sous- 


\section{Mantsanga et al. J. Appl. Biosci. 2016 Effet de l'alimentation calcique séparée sur les performances de production de la poule LHOMAN BROWN sous climat tropical humide}

produits agricoles et agro-industriels qui sont disponibles au Congo. Elles mettent en exergue une valorisation des matières première locales.

\section{REMERCIEMENT}

Ce travail a été réalisé grâce à l'appui financier du Fonds de Soutien à l'Agriculture (FSA Congo)

\section{BIBLIOGRAPHIE}

Abdelatif M., Bordas A., Merat P. 1986. Consommation alimentaire chez les poules Fayoumi et Fayoumi $x$ Leghorn en fonction de la formation de l'œuf et de l'oviposition avec ou sans source calcique séparée. Génétique Sélection et Évolution. $18: 63-72$.

Akouango P., 2014. Évolution qualitative et quantitative des composantes de l'œuf pendant les trois phases de ponte chez la poule. Journal of Applied Biosciences, 74, 6080 - 6085

Aviforum 1999. Sélection et Performances des animaux hybrides, 12.

Anonyme 2013. La classification et la numérotation des œufs de poules. En ligne disponible sur, http://www.regime-dietetique.net/dietetiquepratique/article-dietetique-158.aspx, consulté le 18 mars 2015.

Banga-Mboko H., Minvielle F., Leroy PL. 2001. Effects of separate calcium feeding on laying hens selected for low $\left(R^{-}\right)$or high $\left(R^{+}\right)$residual feed consumption. Animal Research, 50: 239-250.

Banga-Mboko H., Bordas A., Minvielle F., Leroy P.L. 2003. Réponse à l'alimentation calcique séparée de poules pondeuse sélectionnées sur la consommation résiduelle. Annales de Médecine Vétérinaire $147: 51-58$

Banga-Mboko H., Mabandza-Mbandza B., Adzona P. P., Batessana C. 2007. Réponse à l'alimentation calcique séparée de lignées commerciales de poules pondeuse shaver dans les conditions tropicales du Congo Brazzaville. Bulletin of Animal Production and Health in Africa, 55: 4350.

Bordas A., et Minveille F. 1997. Réponse à la chaleur de poules pondeuses de lignées sélectionnées pour une faible ( $R$-) ou forte $(\mathrm{R}+)$ consommation alimentaire résiduelle. Génetic. Selection Évolution. 29(2), 279-290.

Brou G. K. G., Houndonougbo F. M., Aboh A. B., Mensah G. A., Fantodji A. 2012. Effet de la variation temporelle de la température ambiante journalière sur le poids des œufs de poules pondeuses ISA Brown en Côte-d'Ivoire.
International Journal of Chemical and Biological Sciences, 6(5) : 2158-2169.

Cabrera M.C., Sauveur B., Mongin P. 1982. Effect of separate calcium feeding and a limited feeding program on the metabolizable energy of the diet and on nitrogen, calcium and phosphorus retention in laying hens. Reproduction. Nutrition and Développement, 22, 973-987

FAO, 2008. Importance et perspective du secteur avicole au Burkina Faso. Revue du secteur avicole, 43 $p$.

FAO, 2009. Analyse et cartographie de la filière avicole. In: cartographie des filières d'élevages au Congo Brazzaville, FAO, 10-25pp.

Joly P. 1999. Facteurs influençant le poids de l'œuf: comment l'adapter au besoin du marché ? Bulletin du Service Technique, 21p.

Mialoundama F. et Ndinga P., 2006. Adaptabilité des cultivars à la variabilité et aux changements climatiques en République du Congo. Programme des Nations Unies pour le Développement (PNUD), Brazzaville, $59 \mathrm{p}$.

Mongin P., et sauveur B. 1975. Données et application nouvelles concernant la solidité de la coquille et l'œuf .Alimentation calcique séparée .Courrier Avicole, 553(1) ,6-9.

OFIVAL, 2005. Note technique sur la filière avicole. En ligne, www.office-élevage.fr/consulté le 12 février 2012, 20p.

Picard M., Sauveur B., Fenardji F., Angulo I., Mongin P. 1993. Ajustements technico-économiques possibles de l'alimentation des volailles dans les pays chauds. Productions Animales 2:87-103.

Samba G. et Moundza P., 2007. Brazzaville, croissance urbaine et problèmes environnementaux. Centre de recherches sur les tropiques humides, Université Marien Ngouabi-Congo, 15p

Sauveur B., Picard M. 1990. Effets de la température et de l'éclairement appliqués à la poule sur la qualité de l'œuf. Options Méditerranéennes, 7,16 . 